\title{
Crystal field and magnetism with Wannier functions: Rare-earth doped aluminum garnets.
}

\author{
E. Mihóková*, P. Novák, and V. V. Laguta \\ Institute of Physics, Acad. of Sciences of the Czech Rep., \\ Cukrovarnická 10, 162 53 Prague 6, Czech Republic
}

(Dated: May 1, 2022)

\begin{abstract}
Using the recently developed method we calculate the crystal field parameters in yttrium and lutetium aluminum garnets doped with seven trivalent Kramers rare-earth ions. We then insert calculated parameters into the atomic-like Hamiltonian taking into account the electron-electron, spin-orbit and Zeeman interactions and determine the multiplet splitting by the crystal field as well as magnetic $\hat{g}$ tensors. We compare calculated results with available experimental data.
\end{abstract}

\footnotetext{
* Fax:+420 2312 3184, e-mail: mihokova@fzu.cz
} 


\section{INTRODUCTION}

To explain certain optical and magnetic properties of rare-earth $(R)$ materials, determination of crystal field parameters (CFP) is essential. When sufficient experimental data are available CFP are usually determined by the least squares fit. Since the number of nonzero CFP depends on the site symmetry and may be as high as 27 , such method often ends up being ambiguous. As a result, there has been a continuous effort to estimate CFP theoretically (for the review of various methods, see Ref. [1, 2]).

Recently, a novel theoretical approach to calculate CFP has been proposed [3]. An original motivation of the work was to explain the magnetic properties of rare-earth cobaltites $R \mathrm{CoO}_{3}$ where available experimental data do not suffice to estimate CFP. The method starts with the density functional theory (DFT) based band structure calculation, followed by a transformation of the Bloch to Wannier basis. The local Hamiltonian is then expanded in terms of the spherical tensor operators. Resulting CFP are inserted in an atomic-like Hamiltonian involving the crystal field, $4 f-4 f$ correlation, spin-orbit coupling and Zeeman interaction. The method does not suffer from the $4 f$ electron self-interaction (the difficult problem of DFT ab initio methods). The hybridization of the $4 f$ states with other valence orbitals is taken into account via hybridization parameter $\Delta$, a single parameter of the method. In the recent paper [4] a relatively simple way allowing to estimate this parameter was suggested and applied to the $R: \mathrm{LaF}_{3}$ system.

The method has been extensively tested for rare-earth doped aluminates $\mathrm{YAlO}_{3}: \mathrm{R}^{3+}$ with orthorhombic perovskite structure [3]. Remarkable agreement of calculated and experimental data was achieved. Application to gallates $R \mathrm{GaO}_{3}$ and cobaltites $R \mathrm{CoO}_{3}[5]$ as well as manganites $R \mathrm{MnO}_{3}[6]$ followed. Even in these cases calculations provide a fair agreement with available experimental data.

Rare-earth doped aluminum garnets are widely used as laser materials and scintillators due to which extensive experimental data are available. In recent years magneto-optical properties of some non-Kramers ions in garnet hosts have been studied. High magneto-optical activity observed in some cases is of interest in microwave amplifiers and generators. Magnetooptics of $\mathrm{Tb}^{3+}$ and $\mathrm{Tm}^{3+}$ in $\mathrm{Y}_{3} \mathrm{Al}_{5} \mathrm{O}_{12}$ (YAG) is studied in Refs. 7, 8 and Ref. 9, respectively. Magnetooptics of $\mathrm{Eu}^{3+}$ in various garnets is reported in Refs. 10, 11 and that of $\operatorname{Pr}^{3+}$ in YAG in Ref. 12.

In this work we apply the new CFP method to rare-earth doped aluminum garnets. After reviewing the theory and computational procedure, the next section is devoted to analysis of the problem of determination of the parameter $\Delta$ in more detail compared to Ref. 4 . In the following section we calculate crystals field parameters. We show examples of multiplet splitting by the crystal field. We 
focus on Kramers ions and calculation of their magnetic $g$ factors, yet more crystal-field sensitive quantities, and compare them with experimental data.

\section{THEORETICAL APPROACH AND COMPUTATIONAL DETAILS}

The effective Hamiltonian describing $4 f$ states can be written as

$$
\hat{H}_{e f f}=\hat{H}_{A}+\hat{H}_{Z}+\hat{H}_{C F}
$$

where $\hat{H}_{A}$ is the spherically symmetric, free ion atomic-like Hamiltonian (for details see Ref. 13), while $\hat{H}_{Z}$ and $\hat{H}_{C F}$ are the Zeeman interaction and crystal field Hamiltonian, respectively. In the Wybourne notation [14] $\hat{H}_{C F}$ has the form

$$
\hat{H}_{C F}=\sum_{k=2,4,6} \sum_{q=-k}^{k} B_{q}^{(k)} \hat{C}_{q}^{(k)},
$$

where $\hat{C}_{q}^{(k)}$ is a spherical tensor operator of rank $k$ acting on the $4 f$ electrons of the $R$ ion. The coefficients $B_{q}^{(k)}$ are the crystal field parameters. Hermiticity of $\hat{H}_{C F}$ requires that $\left(B_{-q}^{(k)}\right)^{*}=(-1)^{q} B_{q}^{k}$.

Calculation of crystal field parameters consists of four steps:

1. Standard selfconsistent band calculation with $4 f$ states included in the core. The results yield the crystal field potential, subsequently used in the next step.

2. The $4 f$ as well as oxygen $2 p$ and $2 s$ states are treated as the valence states in a nonselfconsistent calculation, all other states are moved away using the orbital shift operator. Relative position of $4 f$ and oxygen states is adjusted using the hybridization parameter $\Delta$ (a single parameter of the method).

3. The $4 f$ band states are transformed to Wannier basis using the wien2wannier [15] and wannier90 [16] packages.

4. Local $4 f$ Hamiltonian in the Wannier basis is extracted and expanded in a series of spherical tensor operators. The coefficients of expansion are the crystal field parameters.

To perform the band structure calculations in steps 1 and 2 we used the WIEN2k package [17] with implemented augmented plane waves + local orbital method. For the exchange correlation functional we applied the generalized-gradient approximation form [18]. We used experimental lattice parameters of $\mathrm{Y}_{3} \mathrm{Al}_{5} \mathrm{O}_{12}(\mathrm{YAG})$ and $\mathrm{Lu}_{3} \mathrm{Al}_{5} \mathrm{O}_{12}(\mathrm{LuAG})$ [19], but the atomic positions within the 
unit cell were optimized for each $R$ substitution by minimizing the atomic forces. The unit cell in our calculations consisted of 80 atoms. The eigenvalue problem was solved in five points of the irreducible Brillouin zone and the number of basis functions was $\sim 7700$ (corresponding to the parameter $\left.R K_{\max }=6.5\right)$. The calculations were non-spin polarized. The atomic radii of $\mathrm{R}(\mathrm{Y}, \mathrm{Lu})$, $\mathrm{Al}$ and $\mathrm{O}$ were 2.3, 1.7 and 1.55 , respectively.

Once the crystal field parameters were determined we used the modified lanthanide package [20] to solve the eigenproblem for the Hamiltonian (1). The results provide the multiplet splitting by the the crystal field. From the energy dependence on external magnetic field one extracts the $\hat{g}$ tensor (for more details see Refs. 3 , $\underline{5}$ ).

\section{HYBRIDIZATION PARAMETER}

The parameter $\Delta$ appears due to hybridization between the rare-earth $4 f$ states and the valence states of its ligands. Our treatment of hybridization is briefly described in Ref. 3 . In the $R$ containing orthorhombic perovskites [3, 5, 6] remarkable agreement between experimentally obtained spectroscopic data and the calculation was obtained by fixing the value of $\Delta$ at 0.6 Ry. With the same $\Delta$ also the magnetism was calculated and compared with experiment. However, less experimental data were available and the agreement, though still satisfactory, was not as good as in case of spectroscopy. In $R: \mathrm{LaF}_{3}$ very good agreement between optical data and calculation was obtained for $\Delta=0.4 \mathrm{Ry}[4]$. In the same paper $\Delta$ was estimated using a charge transfer energy

$$
\Delta \simeq E_{t o t}\left(4 f^{(n+1)}, N_{v a l}-1\right)-E_{t o t}\left(4 f^{n}, N_{v a l}\right)
$$

where $E_{\text {tot }}\left(4 f^{n}, N_{v a l}\right)$ is the total energy of the ground state of the system ( $n_{4 f}$ electrons in $4 f$ shell of R ion and $N_{v a l}$ electrons in the valence band), while $E_{t o t}\left(4 f^{(n+1)}, N_{v a l}-1\right)$ corresponds to the excited state in which one of the valence electrons was transferred in the $4 f$ electron shell. The hybridization parameter thus can be calculated by performing two calculations with $4 f$ electrons treated as the core states - the first one with $4 f^{n}, N_{v a l}$, the second with $4 f^{(n+1)}, N_{v a l}-1$ electron configurations.

Using the above equation we calculated $\Delta$ for the $R$ in question in both YAG and LuAG. The results, together with the data for $R: \mathrm{LaF}_{3}$ and orthorhombic perovskites $R \mathrm{MnO}_{3}$, are shown in Fig. 11.

There are several problems connected with the above method. The first one is connected with the multiplet splitting of the $4 f$ levels, which is not provided by the DFT calculation. In principle this splitting may be obtained by the atomic-like program, we are using. However, there is a problem of double counting of the electron-electron correlation, which would be difficult to overcome. We can 


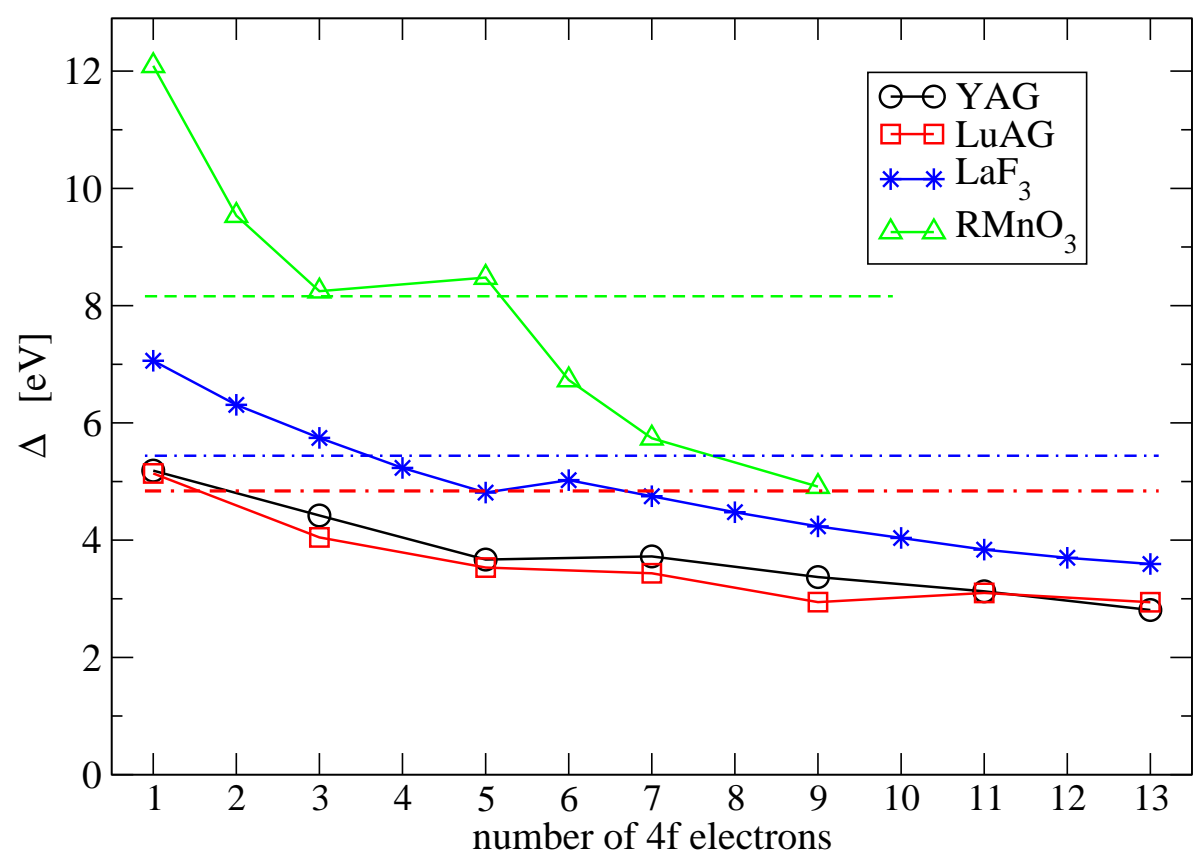

FIG. 1: Dependence of the hybridization parameter $\Delta$ on number of the $4 f$ electrons calculated using eq. 3. Dashed and dash-and-dotted lines correspond to the $\Delta$ values, which were adopted for calculations in $R \mathrm{MnO}_{3}$ and $R: \mathrm{LaF}_{3}$, respectively [4, 6].

only estimate that corresponding uncertainty of $\Delta$ is on the order of the crystal field splitting i.e. less than $\sim 0.1 \mathrm{eV}$ and it will change with $R$. Such scatter will become relatively more important in $R:$ YAG and $R: \mathrm{LuAG}$ compared to $R: \mathrm{LaF}_{3}$ and $R \mathrm{MnO}_{3}$, because $\Delta$ calculated from (3) is smaller in garnets (cf. Fig. 1).

The second problem is inherent to the open core calculations. Even though the $4 f$ electrons are well localized, small part of their density leaks out of the $R$ atomic sphere. Understandably, this leakage is bigger for the $4 f^{(n+1)}$ electron configuration. For $R$ :LuAG we calculated $\Delta$ for three values of the atomic sphere radius $R_{M T}$. The result is shown in Fig. 2 ,

Finally, note that eq. (3) is based on the first order perturbation theory [3], thus higher order correction is needed if the hybridization is important.

\section{RESULTS}

\section{A. CFP and multiplet splitting}

Yttrium (Lutetium) aluminum garnets have a cubic structure belonging to the Ia3d space group. The unit cell contains eight molecular units $\mathrm{Y}_{3} \mathrm{Al}_{5} \mathrm{O}_{12}$ (YAG) or $\mathrm{Lu}_{3} \mathrm{Al}_{5} \mathrm{O}_{12}(\mathrm{LuAG})$. The $R$ impurities 


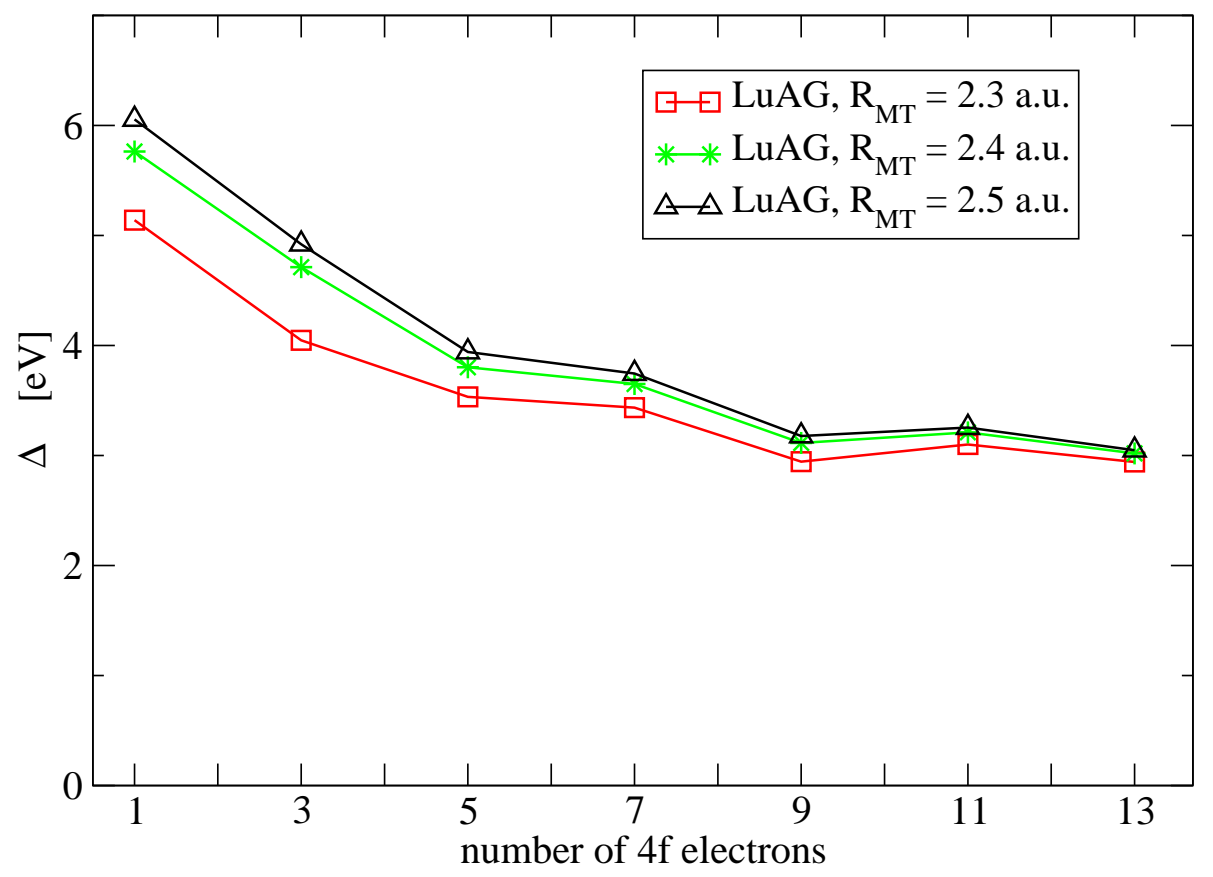

FIG. 2: $R$ :LuAG. Hybridization parameter calculated for three values of the atomic sphere radius $R_{M T}$.

are located on dodecahedrally coordinated Y $(\mathrm{Lu})$ sites of $D_{2}$ symmetry, and nine real parameters are necessary to characterize the crystal field. For the dodecahedral sites, there are six possible coordinate system orientations, which result in six different (but spectroscopically indistinguishable) crystal field parametrizations. The coordinate system to which our CFP and $\hat{g}$ tensors are referred is shown in Fig. 3.

The magnitude of parameter $\Delta$ entering the calculation in the second step was varied between 2.7-10.9 eV (0.2-0.8 Ry) with the step $1.36 \mathrm{eV}(0.1 \mathrm{Ry})$. As $\Delta$ decreases, the $4 f$ levels get closer to the valence band, for $\Delta=2.7 \mathrm{eV}$ the calculation becomes less stable for lighter $R$ and it crashes for Er and Yb. Similarly as in orthorhombic perovskites [3, 5, 6] and $\mathrm{LaF}_{3}[4]$ for fixed $\Delta$ the CFP change smoothly with the number of $4 f$ electrons. In Fig. 4 this is documented for $\Delta=5.4 \mathrm{eV}$ and YAG. As a function of the hybridization parameter, CFP also change smoothly. An example for Sm:YAG is displayed in Fig. 5. The CFP for both YAG and LuAG and all seven $R$ are collected in Table IVA. They were calculated taking $\Delta=5.4 \mathrm{eV}$, which is the lowest value for which the calculation runs smoothly for all $R$.

With the knowledge of CFP, modified program 'lanthanide' [20] was used to calculate the energy levels. Parameters of the free ion Hamiltonian were taken from Ref. 13. The agreement between theory and experiment is generally fairly good. Three examples, namely $\mathrm{Nd}^{3+}, \mathrm{Sm}^{3+}$ and $\mathrm{Er}^{3+}$ in YAG are shown in Figs. 6, 7, 8, For lighter $R$ the position of energy levels only slightly depends on 


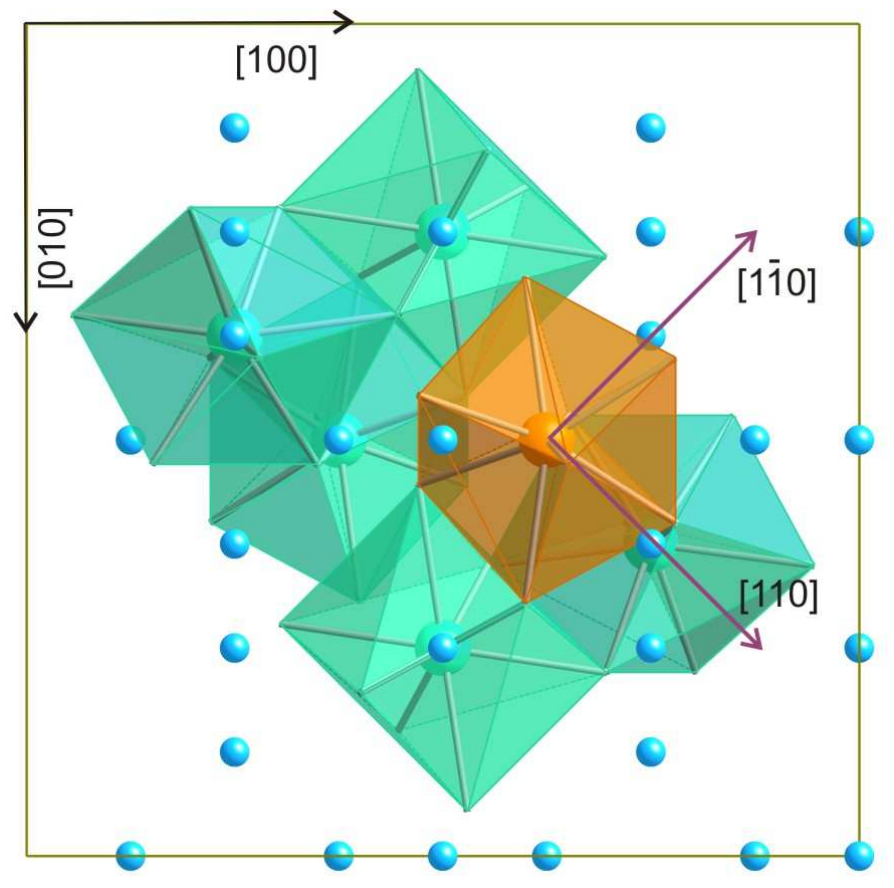

FIG. 3: YAG structure with the highlighted dodecahedrally coordinated site. Axes $a$ and $b$ are parallel to [11̄0] and [110] directions, axis $c$ is parallel to [001] direction.

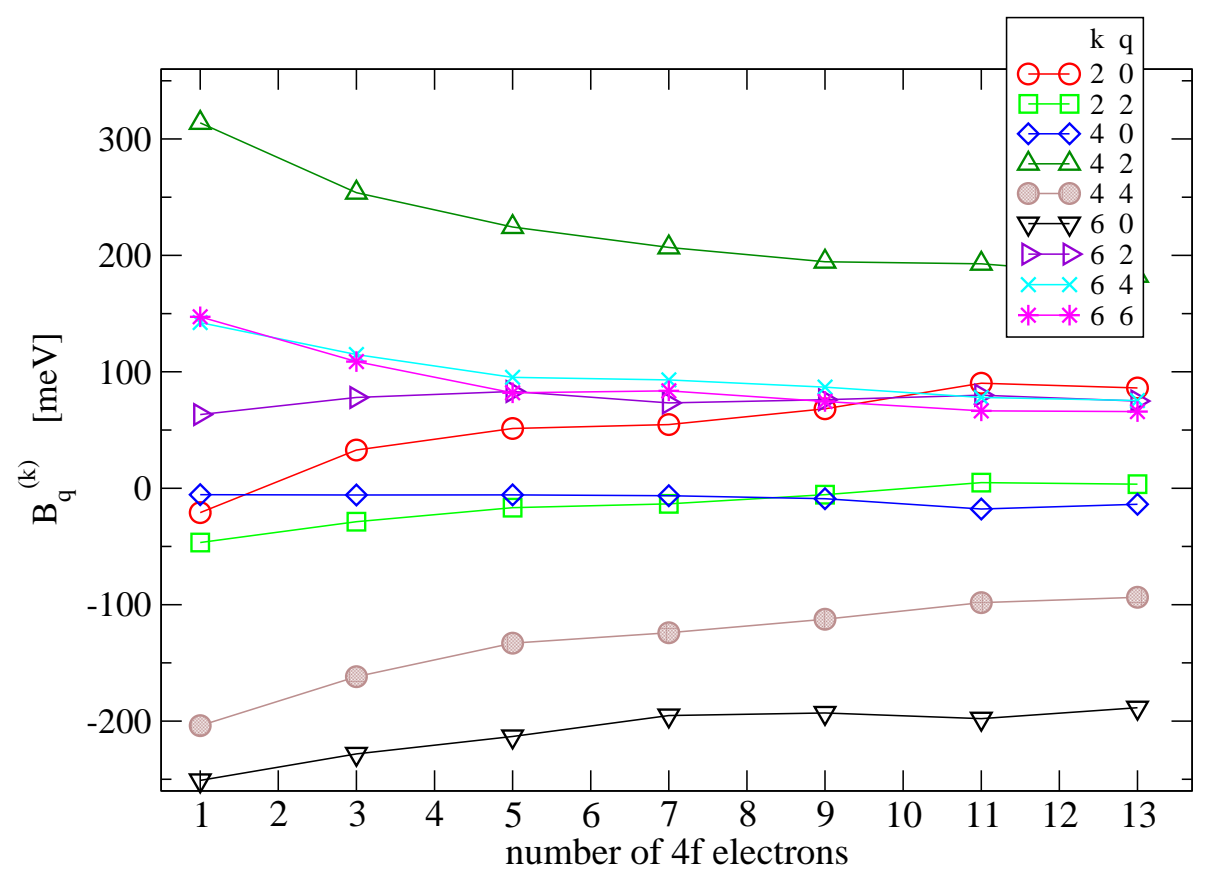

FIG. 4: YAG. CFP as a function of the number of $4 f$ electrons for hybridization parameter $\Delta=5.4 \mathrm{eV}$. 


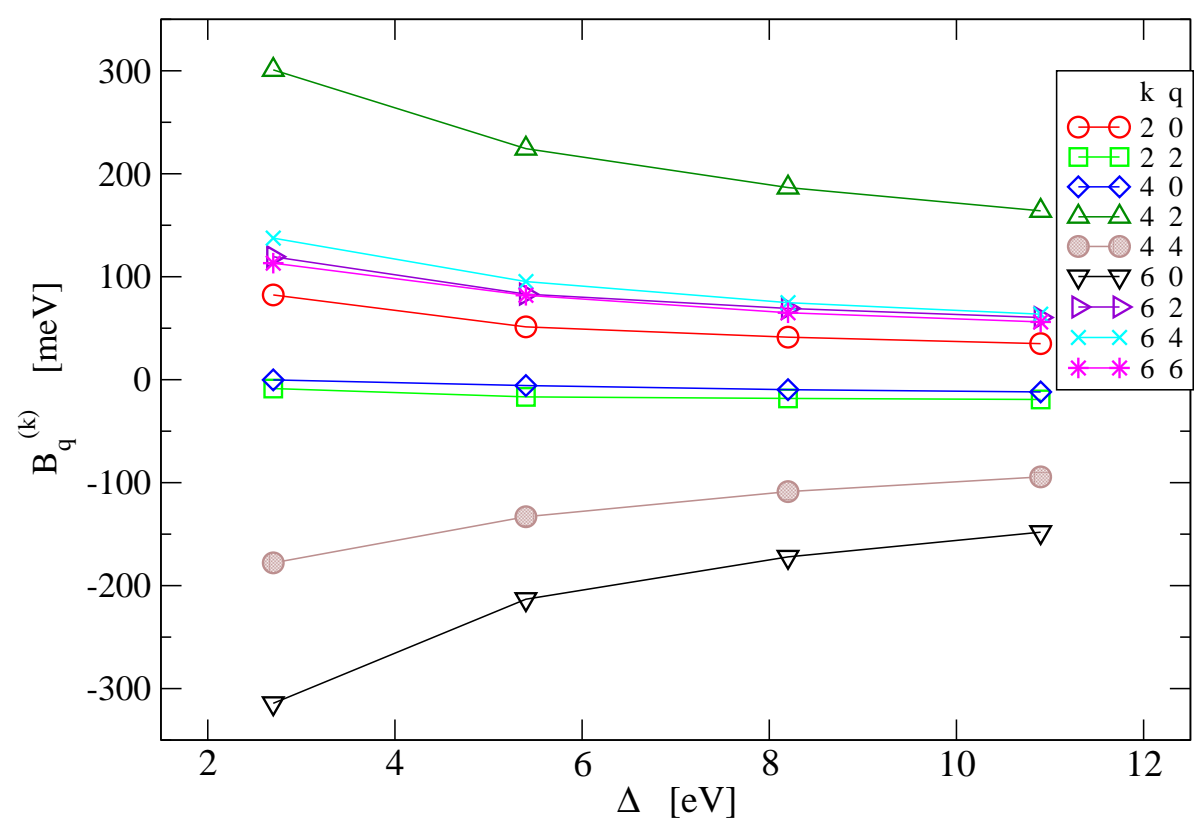

FIG. 5: Sm:YAG. CFP as a function of the hybridization parameter $\Delta$.

the hybridization parameter, for heavier $R$ the dependence becomes stronger [5].

\section{B. $\hat{g}$ tensors}

Axes $a, b, c$ of the system are principal axes of $\hat{g}$. In the experiment components $g_{a}, g_{b}, g_{c}$ are usually determined by analysis of the dependence of EPR spectra on the direction of external magnetic field. There are two sites $R_{1}, R_{2}$ with the $c$ axis running along the [001] direction. Axes $a_{1}, b_{1}$, are parallel to [1̄ㅣ] and [110], respectively. Axes $a_{2}, b_{2}$ are obtained from $a_{1}, b_{1}$ by a $\pi / 2$ rotation around $c$. This leads to an ambiguity in assessment of $g_{a}, g_{b}$ to $R_{1}, R_{2}$ sites [24]. On the other hand, in the calculation the assessment is unambiguous and it refers to the $R$ site shown in Fig. 3,

To determine $\hat{g}$ the effective Hamiltonian (1) was diagonalized with gradually increasing external magnetic field $B$. Resulting dependence of energies was then expanded up to the second power of $B$. The linear term provides $\hat{g}$. Comparing to energies in the zero-field, $\hat{g}$ is much more susceptible to parameters of the calculation, in particular to $\Delta$. In Figs. 9-15 the dependence $\hat{g}(\Delta)$ is shown for all $R$ in YAG and LuAG. As mentioned above, for small $\Delta$ the calculations become unreliable, which results in fluctuations in $\hat{g}(\Delta)$ dependence. The calculation for $\Delta=5.4 \mathrm{eV}$ and $R: Y A G, R: L u A G$ is compared with the experiment in Tables $\amalg$ and 【II. 
TABLE I: Crystal field parameters (in meV) in $R$ :YAG and $R: L u A G$ calculated for hybridization parameter $\Delta=5.4 \mathrm{eV}$.

\begin{tabular}{|c|c|c|c|c|c|c|c|}
\hline k q & Ce:YAG & Nd:YAG & Sm:YAG & Gd:YAG & Dy:YAG & Er:YAG & Yb:YAG \\
\hline 20 & -21.2 & 32.8 & 37.0 & 52.4 & 67.4 & 113.1 & 72.2 \\
\hline 22 & -46.6 & -28.7 & -23.8 & -14.4 & -5.6 & 15.4 & 2.1 \\
\hline 40 & -5.6 & -5.8 & -1.1 & -5.8 & -8.6 & -22.9 & -3.0 \\
\hline 42 & 313.8 & 253.9 & 223.2 & 206.3 & 193.1 & 183.8 & 163.6 \\
\hline 44 & -203.9 & -161.9 & -139.5 & -125.7 & -114.4 & -87.3 & -88.0 \\
\hline 60 & -250.8 & -228.2 & -203.9 & -193.4 & -192.4 & -207.8 & -188.3 \\
\hline 62 & 63.0 & 77.9 & 73.8 & 71.1 & 75.2 & 93.4 & 80.4 \\
\hline 64 & 142.4 & 114.9 & 102.4 & 94.6 & 88.5 & 68.3 & 67.1 \\
\hline 66 & 147.5 & 108.8 & 95.2 & 86.2 & 75.8 & 54.1 & 53.9 \\
\hline \multicolumn{8}{|c|}{ Ce:LuAG Nd:LuAG Sm:LuAG Gd:LuAG Dy:LuAG Er:LuAG Yb:LuAG } \\
\hline 20 & -36.3 & 22.7 & 33.8 & 43.7 & 52.2 & 80.9 & 77.1 \\
\hline 22 & -33.0 & -20.4 & -14.2 & -9.1 & -3.5 & 18.8 & 17.8 \\
\hline 40 & 4.6 & 1.9 & -2.1 & -2.0 & -1.8 & -28.9 & -27.1 \\
\hline 42 & 320.4 & 255.8 & 209.1 & 184.5 & 165.6 & 188.6 & 164.5 \\
\hline 44 & -207.8 & -165.1 & -132.0 & -114.5 & -105.3 & -97.1 & -83.6 \\
\hline 60 & -252.2 & -229.1 & -197.8 & -178.8 & -169.6 & -210.4 & -185.5 \\
\hline 62 & 67.6 & 81.3 & 73.9 & 68.8 & 68.3 & 79.1 & 69.0 \\
\hline 64 & 141.7 & 118.7 & 97.0 & 84.7 & 78.5 & 71.9 & 60.9 \\
\hline 66 & 148.8 & 109.0 & 89.6 & 75.9 & 66.2 & 57.8 & 50.5 \\
\hline
\end{tabular}

\section{DISCUSSION}

As seen in Fig. 1, the hybridization parameter $\Delta$ for $R$ impurities in YAG and LuAG comes out smaller than in manganites and $\mathrm{LaF}_{3}$. This represents a serious obstacle when trying to get the best $\mathrm{CFP}$, as for $\Delta$ smaller than $\simeq 4 \mathrm{eV}$ the calculation does not always yield reliable results. We traced the problem to the wannier90 package. Calculation with wannier90 provides the maximally localized Wannier functions, but it does not guarantee that they will be centered on the crystal site of the $R$ impurity. Indeed, for $\Delta \simeq 4 \mathrm{eV}$ the functions are displaced for heavier $R$ and for still smaller $\Delta$ the displacement appears for all $R$. As a consequence the symmetry is lost, all CFP are nonzero and for $q \neq 0$ they are complex. A possible remedy may be to use recently proposed scheme by Sakuma 


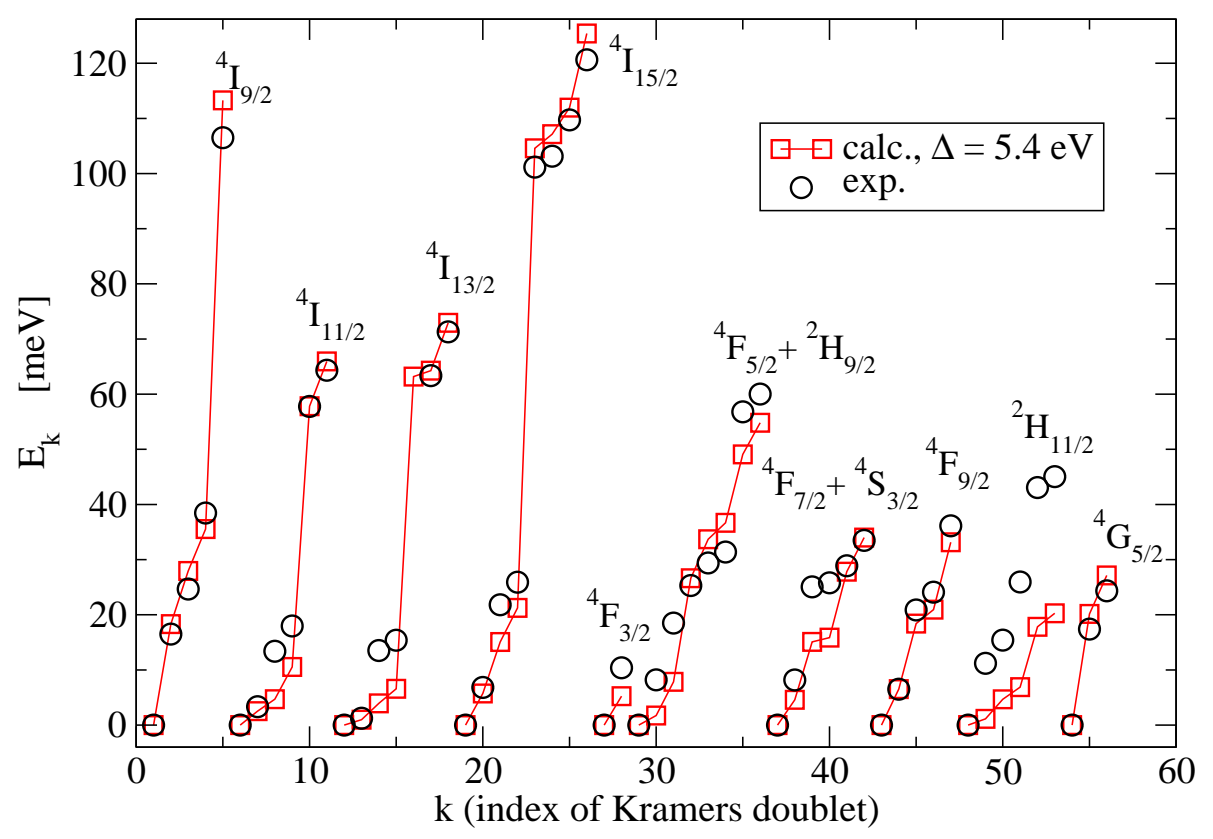

FIG. 6: Nd:YAG. Splitting of the lowest ten multiplets by the crystal field. The solid line is only to guide the eye. The lowest energy of the $j$-th multiplet $(j=1, \ldots, 10)$ for calculated (experimental) results was reduced by 0(0), 255(248), 495(489), 722(714), 1452(1418), 1567(1533), 1694(1656), 1851(1813), 1999(1952), 2020(2088) meV, respectively. Experimental data were taken from Ref. 21.

[32] (symmetry adapted Wannier functions) or simpler, but less sophisticated method of selectively localized Wannier functions [33].

Despite the problem with $\Delta$ the agreement between calculated and experimental multiplet splitting is very good as shown in Figs. 6],7/8, We found similar agreement also for other garnet systems.

In the past the CFP of the $R$ impurities in garnets were determined several times using either semiempirical methods or the least squares fit to the optical data. Prominent groups that adopted this approach are those of Gruber and Burdick, which obtained rich optical data and carefully analyzed them using the least squares fit. In Table IV we compare our CFP with those obtained by these groups for Nd:YAG [21], Sm:YAG [22] and Er:YAG [23].

When considering $B_{q}^{(k)}$ one should be aware of the fact that the spectroscopic methods do not differentiate between the six crystallographically equivalent $R$ sites which, however, have different local coordinate system. The local coordinate systems are connected by symmetry operations of the Ia3d space group and to compare different sets of CFP corresponding symmetry operation has to be applied (see e.g. Refs. 23, 34).

In Table IV] are also compared the quantities $S_{k}$, which were introduced by Leavitt [35] and which 


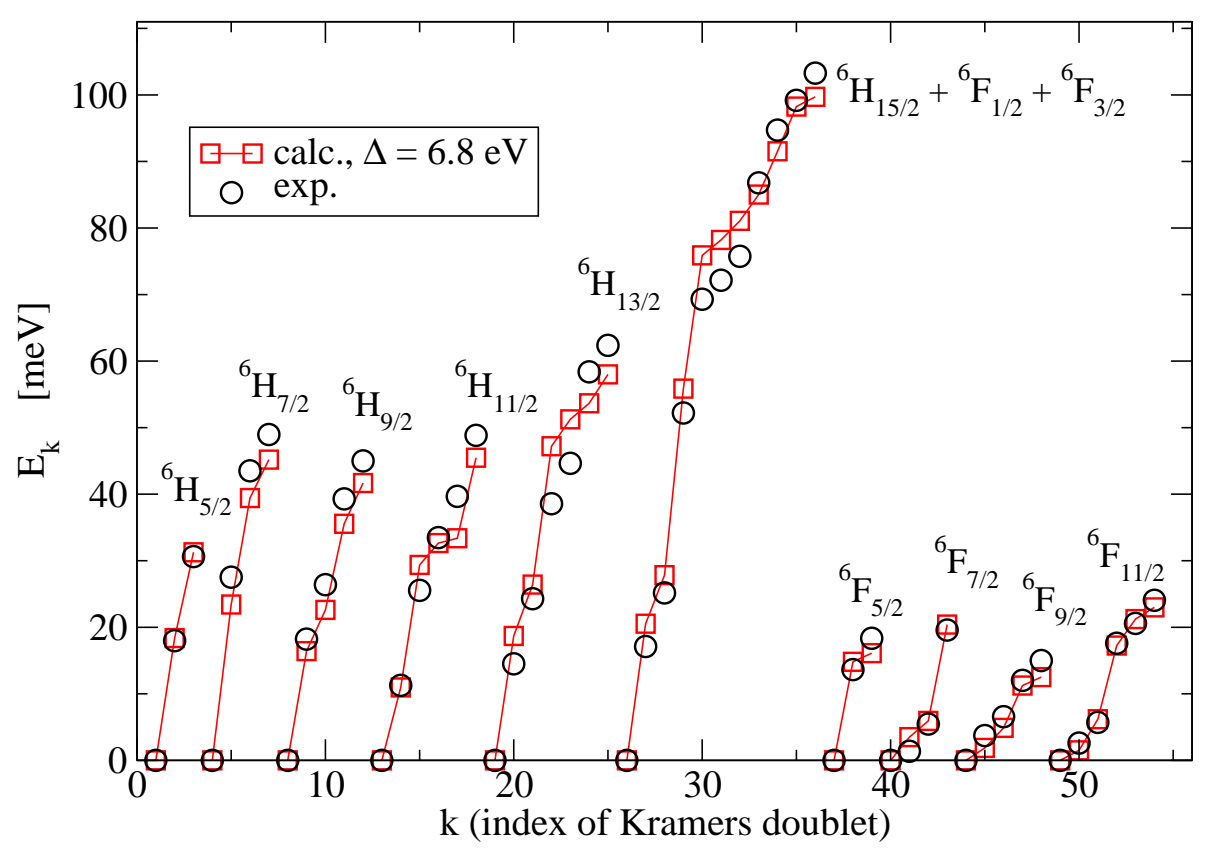

FIG. 7: Sm:YAG. Splitting of the lowest ten multiplets by the crystal field. The solid line is only to guide the eye. The lowest energy of the $j$-th multiplet $(j=1, \ldots, 10)$ was reduced for calculated (experimental) results by 0(0), 129(126), 284(279), 446(440), 609(603), 768(761), 909(813), 1017(831), 1164(833), 1331(848) meV, respectively. Experimental data were taken from Ref. 22.

TABLE II: $\hat{g}$ tensor components of the ground Kramers doublets along principal axes in YAG: ${ }^{3+}$ calculated for hybridization parameter $\Delta=5.4 \mathrm{eV}$, except for Er where $\Delta=4.8 \mathrm{eV}$. Experimental data refer to the work listed in the last column. Difference in per cent between calculated and experimental values is reported.

\begin{tabular}{|c|c|c|c|c|c|c|c|}
\hline$R$ & calc. & $\begin{array}{l}\quad g_{a} \\
\text { exp. diff. }\end{array}$ & calc. & $\begin{array}{l}g_{b} \\
\text { exp. diff. }\end{array}$ & calc. & $\begin{array}{l}g_{c} \\
\text { exp. diff. }\end{array}$ & Ref. \\
\hline $\mathrm{Ce}$ & 1.06 & 0.9116 & 2.54 & 1.8736 & 2.32 & 2.7415 & [26] \\
\hline $\mathrm{Nd}$ & 2.16 & 1.7424 & 1.74 & 1.1650 & 3.52 & 3.9110 & [30] \\
\hline $\mathrm{Sm}$ & 0.59 & $-\quad-$ & 0.13 & $-\quad-$ & 0.02 & $-\quad-$ & - \\
\hline Gd & 1.82 & 1.999 & 1.82 & 1.999 & 2.43 & 1.9922 & [28] \\
\hline Dy & 0.07 & 0.4083 & 0.30 & 0.7359 & 18.9 & 18.24 & [29] \\
\hline Er & 8.41 & 7.759 & 3.77 & 3.712 & 7.50 & 7.352 & [29] \\
\hline $\mathrm{Yb}$ & 3.97 & 3.785 & 3.83 & 3.871 & 2.52 & 2.472 & [25] \\
\hline
\end{tabular}




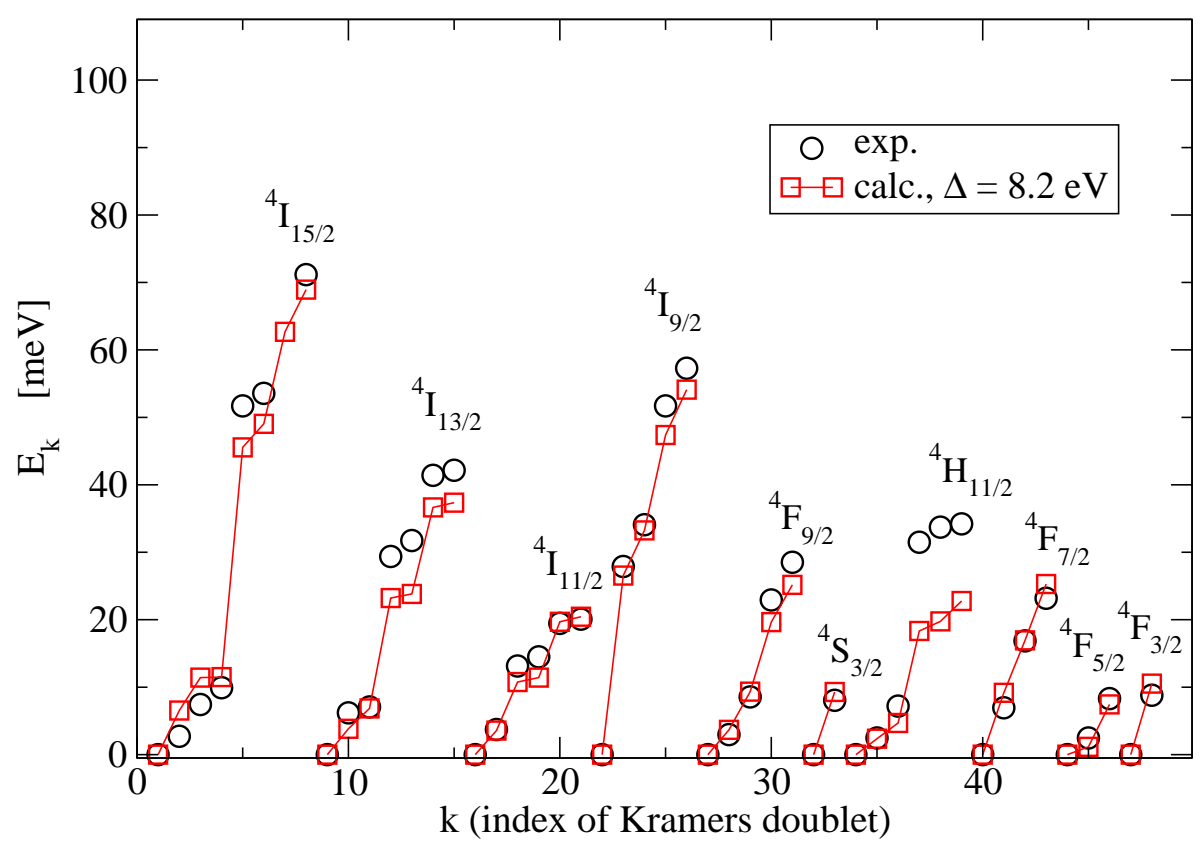

FIG. 8: Er:YAG. Splitting of the lowest ten multiplets by the crystal field. The solid line is only to guide the eye. The lowest energy of the $j$-th multiplet $(j=1, \ldots, 10)$ for calculated (experimental) results was reduced by 0(0), 812(812), 1271(1272), 1525(1525), 1895(1896), 2280(2281), 2367(2370), 2543(2543), 2755(2755), $2801(2803)$

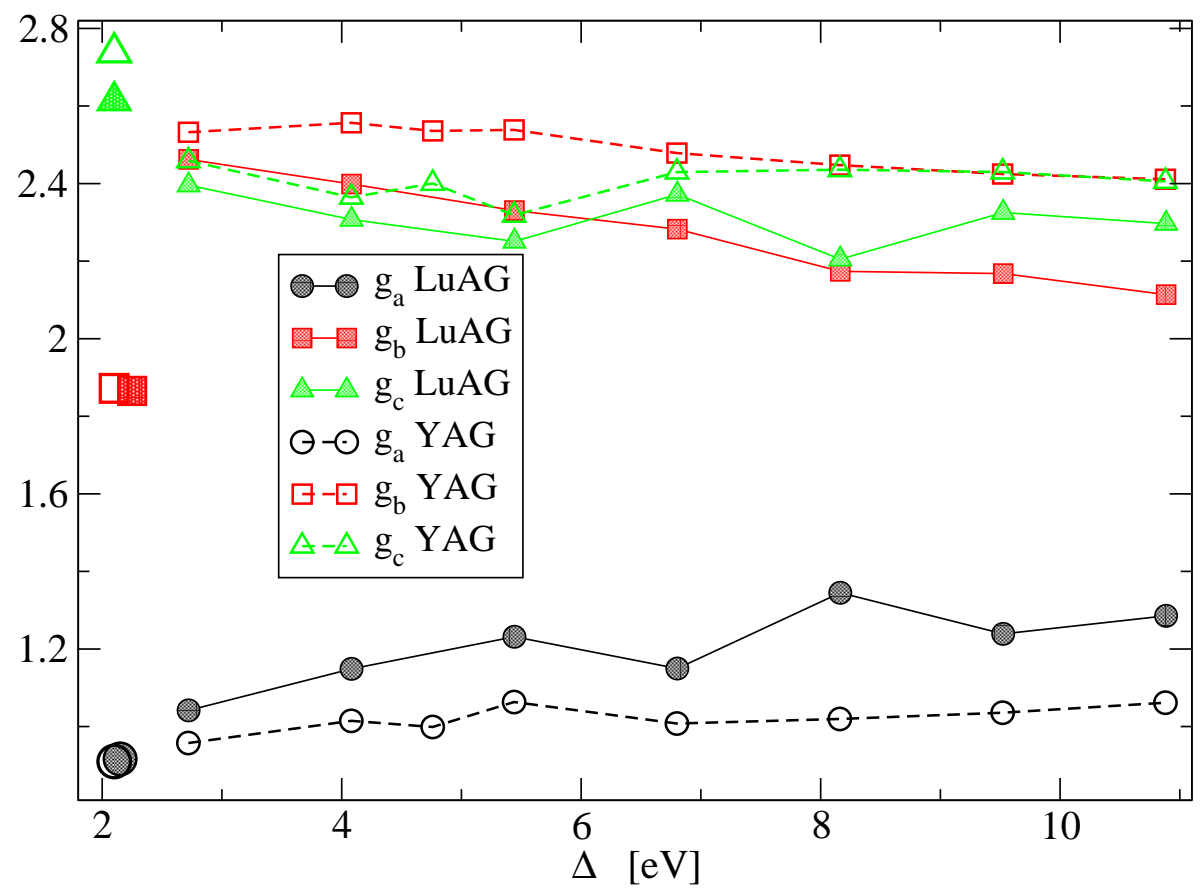

FIG. 9: Ce:YAG, LuAG. Principal components of $\hat{g}$ as a function of the hybridization parameter $\Delta$. Experimental data (enlarged symbols on the left side) are taken from Refs. 26, 27. 


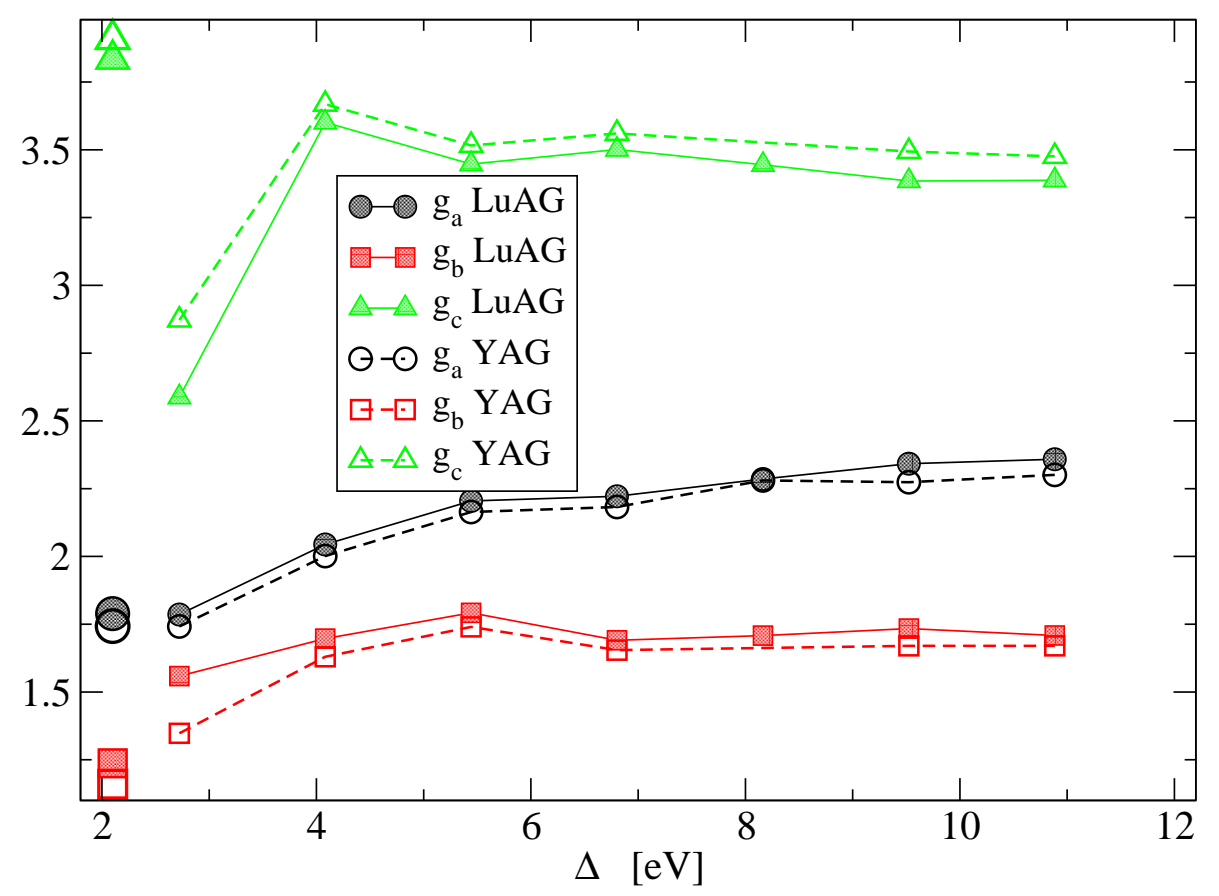

FIG. 10: Nd:YAG, LuAG. Principal components of $\hat{g}$ as a function of the hybridization parameter $\Delta$. Experimental data (enlarged symbols on the left side) are taken from Refs. 24, 30.

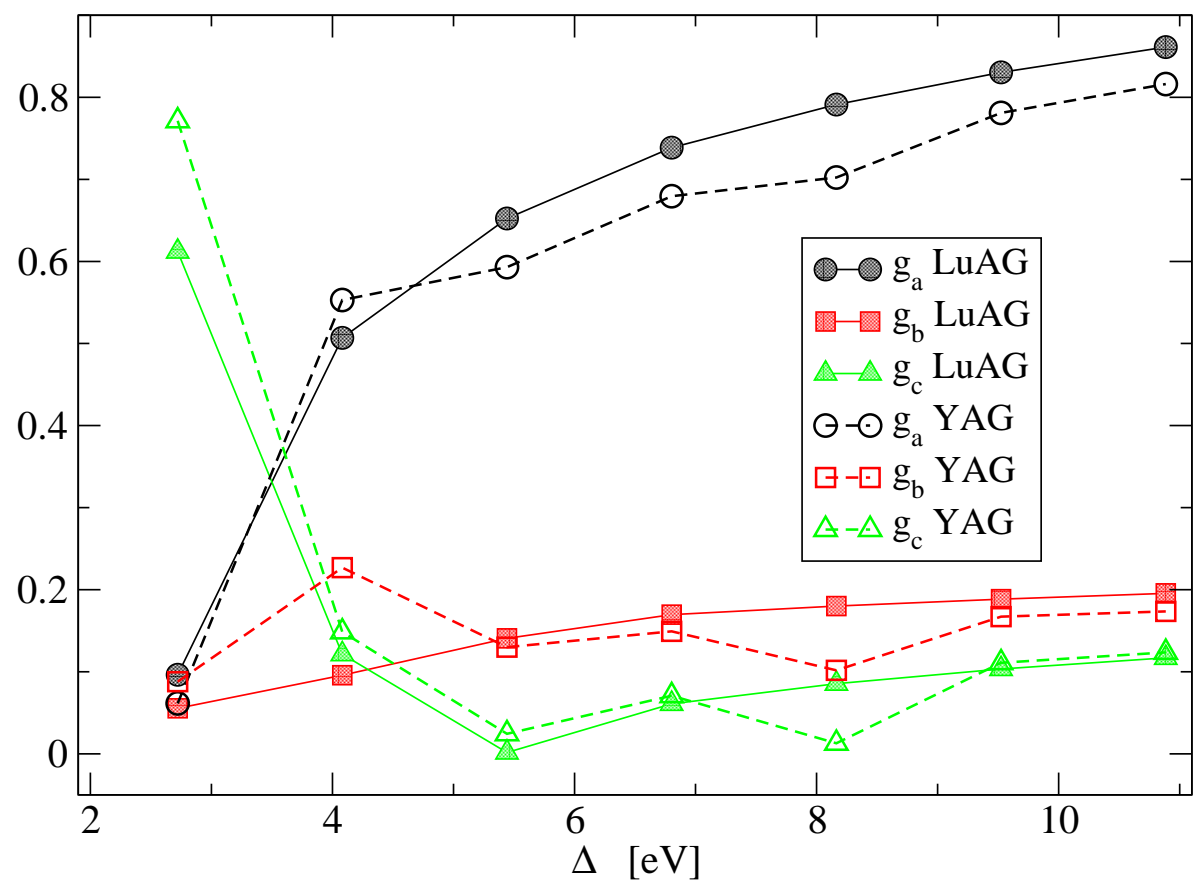

FIG. 11: Sm:YAG, LuAG. Principal components of $\hat{g}$ as a function of the hybridization parameter $\Delta$. Experimental data are not available. 


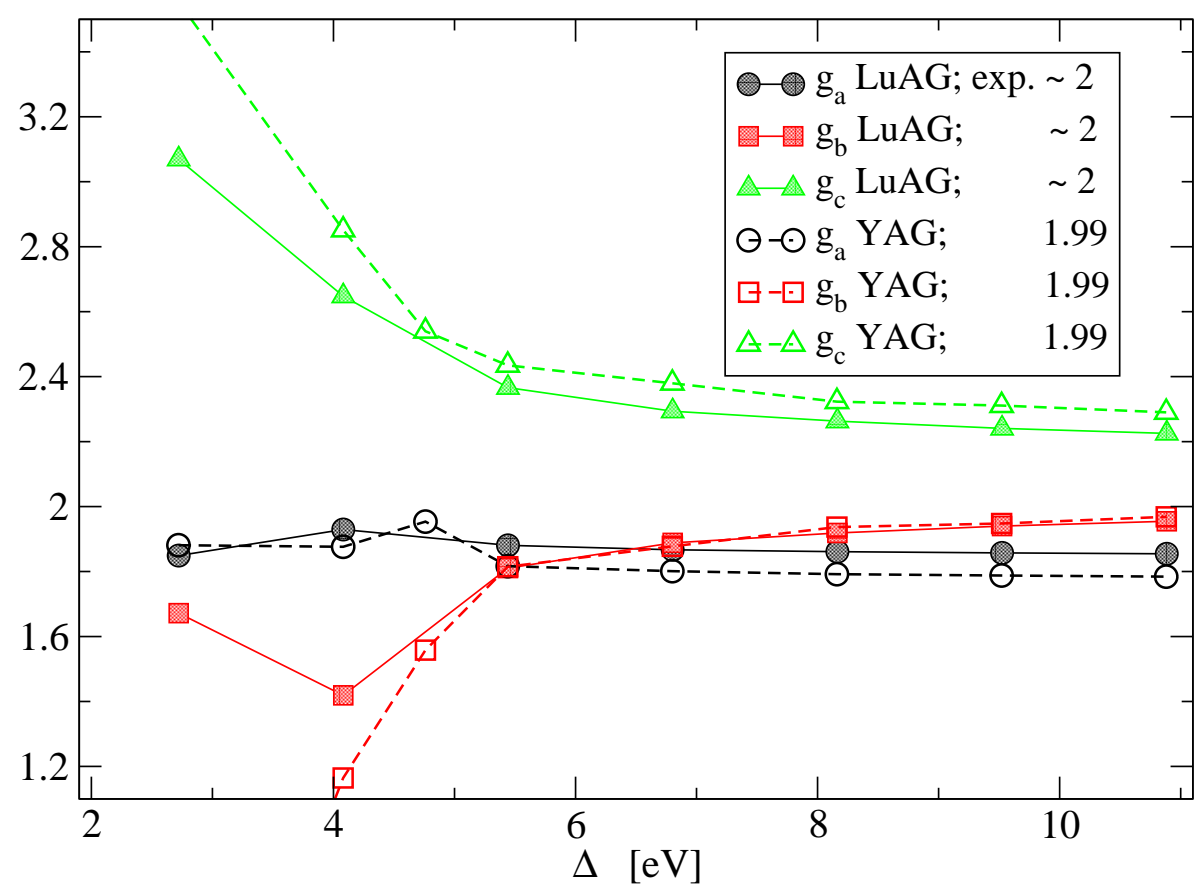

FIG. 12: Gd:YAG, LuAG. Principal components of $\hat{g}$ as a function of the hybridization parameter $\Delta$.

TABLE III: $\hat{g}$ tensor components of the ground Kramers doublets along principal axes in LuAG: $\mathrm{R}^{3+}$ calculated for hybridization parameter $\Delta=5.4 \mathrm{eV}$, except for Er where $\Delta=4.8 \mathrm{eV}$. Experimental data refer to the work listed in the last column. Difference in per cent between calculated and experimental values is reported.

\begin{tabular}{|c|c|c|c|c|c|c|c|}
\hline$R$ & & $\begin{array}{l}g_{a} \\
\text { exp. diff. }\end{array}$ & calc. & $\begin{array}{l}g_{b} \\
\text { exp. diff. }\end{array}$ & & $\begin{array}{l}g_{c} \\
\text { exp. diff }\end{array}$ & Ref. \\
\hline $\mathrm{Ce}$ & 1.23 & 0.9213 & 2.33 & 1.8725 & 2.25 & 2.6114 & [27] \\
\hline $\mathrm{Nd}$ & 2.20 & 1.7923 & 1.79 & 1.2444 & 3.45 & 3.8310 & [24] \\
\hline Sm & 0.65 & $-\quad-$ & 0.14 & $-\quad-$ & 0.002 & - & - \\
\hline $\mathrm{Gd}$ & 1.88 & $-\quad-$ & 1.81 & $-\quad-$ & 2.37 & - & - \\
\hline Dy & 0.55 & 2.2976 & 0.11 & 0.9188 & 18.74 & 16.613 & [29] \\
\hline Er & 6.87 & 4.1267 & 4.01 & 8.4352 & 8.66 & 6.9325 & [29] \\
\hline $\mathrm{Yb}$ & 3.83 & $\begin{array}{ll}3.82 & 0.3\end{array}$ & 4.02 & 3.728 & 2.48 & 2.574 & [31] \\
\hline
\end{tabular}

are invariant with respect to the rotation of the coordinate system:

$$
S_{k}=\left[\frac{1}{2 k+1} \sum_{q=-k}^{k}\left|B_{q}^{(k)}\right|^{2}\right]^{1 / 2} .
$$

We now turn to the magnetism, which is more susceptible to the values of CFP compared to the 


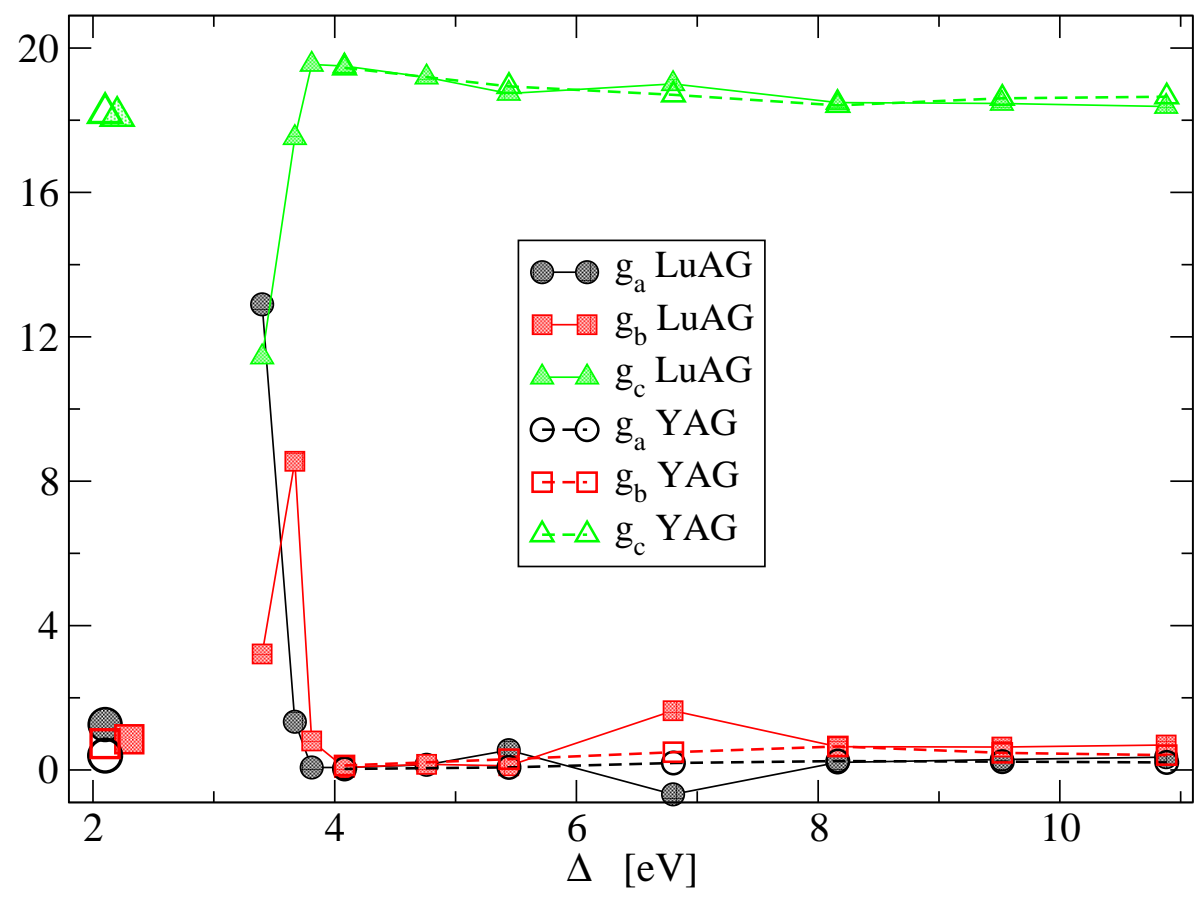

FIG. 13: Dy:YAG, LuAG. Principal components of $\hat{g}$ as a function of the hybridization parameter $\Delta$. Experimental data (enlarged symbols on the left side) are taken from Ref. 29.

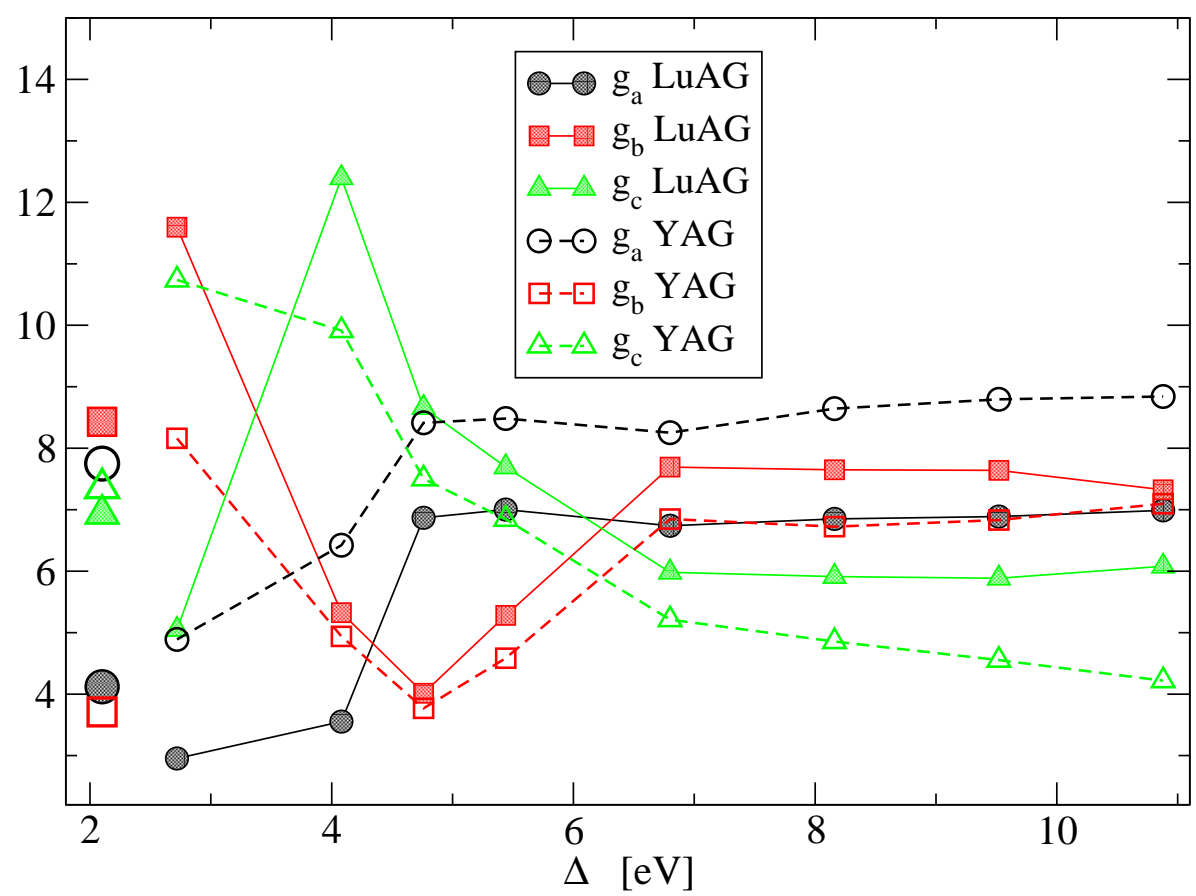

FIG. 14: Er:YAG, LuAG. Principal components of $\hat{g}$ as a function of the hybridization parameter $\Delta$. Experimental data (enlarged symbols on the left side) are taken from Ref. 29. 


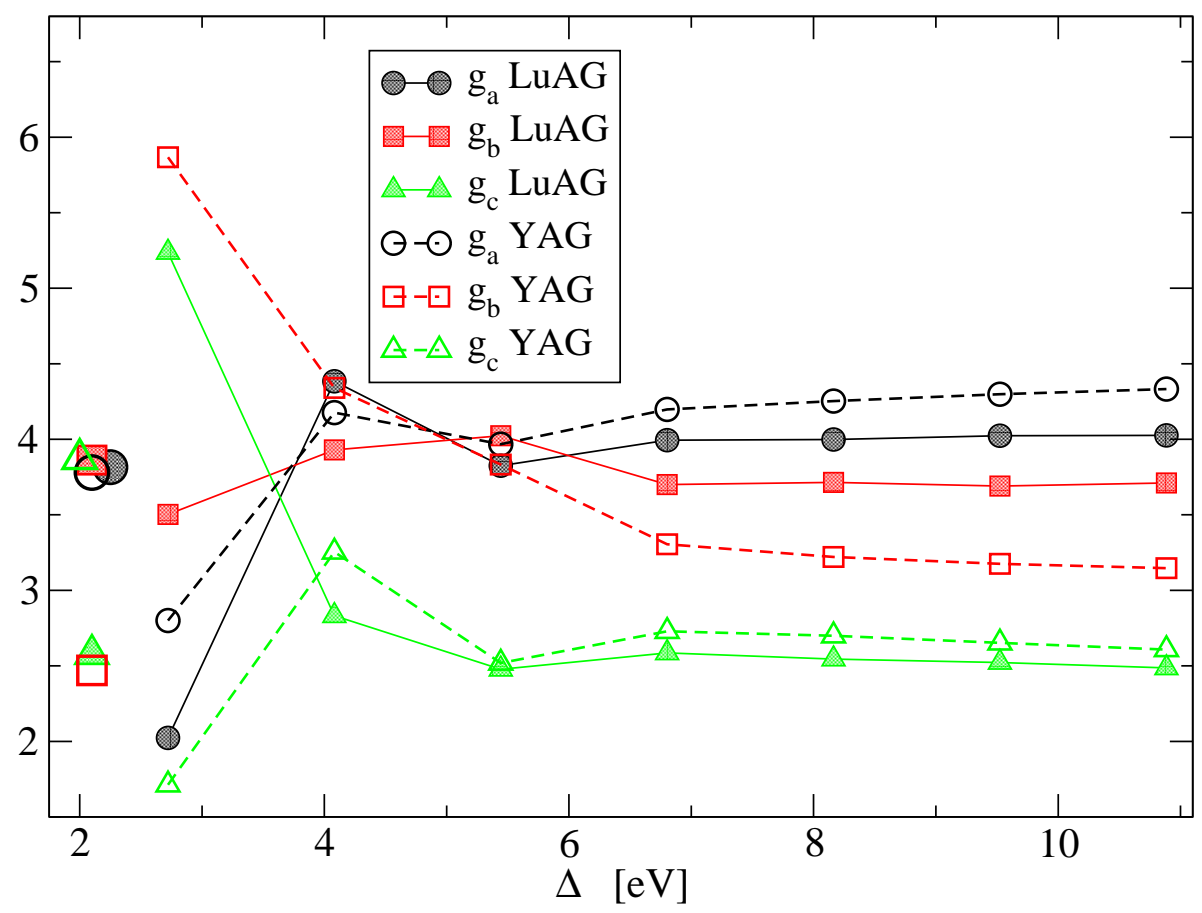

FIG. 15: Yb:YAG, LuAG. Principal components of $\hat{g}$ as a function of the hybridization parameter $\Delta$ Experimental data (enlarged symbols on the left side) are taken from Refs. 25, 31.

energies in the zero magnetic field. Despite this sensitivity and the above mentioned problem with $\Delta$ smaller than $4 \mathrm{eV}$, the calculated $\hat{g}$ tensors qualitatively reflect the experimental data. The method will be thus particularly useful for magnetic, nontransparent rare-earth compounds, in which the experimental data do not allow determination of the crystal field.

Our approach is relatively simple, it may be used by non specialists and corresponding programs, as well as the test example, are available on the WIEN2k web site (www.wien2k.at).

\section{CONCLUSIONS}

The crystal field parameters and the $\hat{g}$ tensors of the ground state were calculated for all seven rare-earth Kramers ions substituted for Y $(\mathrm{Lu})$ in YAG (LuAG). Very good agreement with the spectroscopic data and qualitative agreement with experimental $\hat{g}$ tensors was found. A comparison of calculated CFP with their counterparts obtained by the least squares fit to the optical data is fair and it shows that theory can help to avoid the ambiguity inherent to the least squares approach. 
TABLE IV: Comparison of CFP and the rotational invariants $S_{k}$ obtained in this paper and CFP obtained by Gruber et al. and Burdick et al. (these parameters were rotated in the coordinate system used in this paper as described in the text). CFP and $S_{k}$ are in $\mathrm{meV}$, hybridization parameter equals to $4.76 \mathrm{eV}$ for $\mathrm{Nd}$, $6.8 \mathrm{eV}$ for Sm and Er.

\begin{tabular}{rrrrrrrr}
\hline & \multicolumn{4}{c}{ Nd } & \multicolumn{3}{c}{ Sm } \\
$\mathrm{k}$ & \multicolumn{4}{c}{ q this paper Ref. 21 } & this paper Ref. 22 this paper Ref. 23 \\
\hline 2 & 0 & 32.8 & 52.3 & 37.0 & 54.3 & 113.1 & 42.3 \\
2 & 2 & -28.7 & -19.7 & -23.8 & -13.3 & 15.4 & -27.6 \\
4 & 0 & -5.8 & -23.5 & -1.1 & -16.1 & -22.9 & -21.4 \\
4 & 2 & 253.9 & 273.4 & 223.2 & 238.7 & 183.8 & 185.5 \\
4 & 4 & -161.9 & -119.5 & -139.5 & -60.7 & -87.3 & -52.1 \\
6 & 0 & -228.2 & -241.1 & -203.9 & -197.0 & -207.8 & -146.0 \\
6 & 2 & 77.9 & 73.5 & 73.8 & 85.2 & 93.4 & 40.0 \\
6 & 4 & 114.9 & 103.5 & 102.4 & 117.2 & 68.3 & 65.6 \\
6 & 6 & 108.8 & 72.2 & 95.2 & 85.5 & 54.1 & 64.7 \\
\hline 2 & 23.3 & 26.5 & 22.4 & 25.7 & 51.5 & 25.7 & \\
$S_{k} 4$ & 142.0 & 140.9 & 124.1 & 116.2 & 96.2 & 91.1 & \\
6 & 93.8 & 88.0 & 83.9 & 85.7 & 76.4 & 56.5 & \\
\hline
\end{tabular}

\section{Acknowledgments}

We are indebted to Jan Kuneš, Vladimír Nekvasil and Karel Knížek for helpful discussions. This work was supported by the Czech Science Foundation 13-09876S project.

[1] K. Ogasawara, S. Watanabe, H. Toyoshima, and M. Brik, Handbook on Physics and Chemistry of Rare Earths, vol. 37 (Elsevier, 2007), chapter 231.

[2] P. Novák, Rare Earths: New Research (Nova Science Publishers, Inc., New York, 2013), chapter 7.

[3] P. Novák, K. Knížek, and J. Kuneš, Phys. Rev. B 87, 205139 (2013).

[4] P. Novák, J. Kuneš, and K. Knížek, Opt. Mater. 37, 414 (2014).

[5] P. Novák, K. Knížek, M. Maryško, Z. Jirák, and J. Kuneš, J. Phys.: Condens. Matter 25, 446001 (2013). 
[6] P. Novák, V. Nekvasil, and K. Knížek, J. Magn. Magn. Mater. 358-359, 228 (2014).

[7] J. B. Gruber, D. K. Sardar, K. L. Nash, U. V. Valiev, V. Y. Sokolov, , and S. A. Rakhimov, J. Appl. Phys. 103, 103103 (2008).

[8] U. V. Valiev, J. B. Gruber, G. W. Burdick, A. K. Mukhammadiev, D. Fu, and V. O. Pelenovich, Opt. Mater. 36, 1101 (2014).

[9] U. V. Valiev, J. B. Gruber, I. R. Gapdulkhakov, N. I. Juraeva, A. K. Mukhammadiev, S. A. Rakhimov, and I. S. Édel'man, Opt. Spect. 106, 851 (2009).

[10] U. V. Valiev, J. B. Gruber, D. Fu, V. O. Pelenovich, G. W. Burdick, and M. E. Malysheva, J. Rare Earth 29, 776 (2011).

[11] U. V. Valiev, J. B. Gruber, A. K. Mukhammadiev, V. O. Pelenovich, D. Fu, and G. W. Burdick, J. Rare Earth 31, 837 (2013).

[12] U. V. Valiev, J. B. Gruber, G. W. Burdick, A. K. Mukhammadiev, D. Fu, and V. O. Pelenovich, J. Lumin. 145, 393 (2014).

[13] W. T. Carnall, G. L. Goodman, K. Rajnak, and R. S. Rana, J. Chem. Phys. 90, 3443 (1989).

[14] B. G. Wybourne, Spectroscopic properties of rare earth (Interscience, New York, 1965).

[15] J. Kuneš, R. Arita, P. Wissgott, A. Toschi, H. Ikeda, and K. Held, Comput. Phys. Commun. 181, 1888 (2010).

[16] A. A. Mostofi, J. R. Yates, Y.-S. Lee, I. Souza, D. Vanderbilt, and N. Marzari, Comput. Phys. Commun. 178, 685 (2008).

[17] P. Blaha, K. Schwarz, G. K. H. Madsen, D. Kvasnicka, and J. Luitz, Wien2k, An Augmented Plane Wave + Local Orbitals Program for Calculating Crystal Properties

(Technische Universität, Wien, Austria, 2001), iSBN 3-9501031-1-2.

[18] J. P. Perdew, K. Burke, and M. Ernzerhof, Phys. Rev. Lett. 77, 3865 (1996).

[19] Y. Kuwano, K. Suda, N. Ishizawa, and T. Yamada, J. Cryst. Growth 260, 159 (2004).

[20] S. Edwardsson and D. Aberg, Comput. Phys. Commun. 133, 396 (2001).

[21] G. W. Burdick, C. K. Jayasankar, and F. S. Richardson, Phys. Rev. B 50, 16309 (1994).

[22] J. B. Gruber, B. Zandi, and M. F. Reid, Phys. Rev. B 60, 15643 (1999).

[23] G. W. Burdick, J. B. Gruber, K. L. Nash, S. Chandra, and D. K. Sardar, Spectroscopy Letters 43, 406 (2010).

[24] W. Wolf, M. Ball, M. H. M. Leask, and A. Wyatt., J. Phys. Soc. Japan 17, Supp. B-1, 443 (1962).

[25] J. Carson and R. White, J. Appl. Phys. 31, S53 (1960).

[26] H. Lewis, J. Appl. Phys. 37, 739 (1966). 
[27] A. Vedda, M. Martini, D. Martino, V. Laguta, M. Nikl, E. Mihokova, J. Rosa, K. Nejezchleb, and K. Blazek, Rad. Eff. Def. in Solids 157, 1003 (2002).

[28] L. Rimai and G. DeMars, J. Appl. Phys. 33, 1254 (1962).

[29] J. C.P. Poole and H. Farach, CRC Handbook of Spectroscopy (CRC, Boca Raton, FL, 1974).

[30] E. Nikolova and B. Timan, J. Appl. Spectroscopy 36, 348 (1982).

[31] V. Laguta, A. Slipenyuk, M. Glinchuk, M. Nikl, J. Rosa, A. Vedda, and K. Nejezchleb, Opt. Mater. 30, 79 (2007).

[32] R. Sakuma, Phys. Rev. B 87, 235109 (2013).

[33] R. Wang, E. Lazar, H. Park, A. Millis, and C. Marianetti, Phys. Rev. B 90, 165125 (2014).

[34] C. A. Morrison and R. P. Leavitt, Handbook on the physics and chemistry of rare earths, vol. 5, p.632 (North-Holland publishing company, Amsterdam, New York, Oxford, 1982).

[35] R. Leavitt, J. Chem. Phys. 77, 1661 (1982). 